\title{
Near-Infrared Light-Driven Controllable Motions of Gold-Hollow-Microcone Array
}

Hongxu Chen, ${ }^{1}$ Qilong Zhao, ${ }^{1}$ Yunlong Wang, ${ }^{1}$ Shilin Mu, ${ }^{2}$ Huanqing Cui, ${ }^{1}$ Juan Wang, ${ }^{1}$ Tengfei Kong, ${ }^{1}$ Xuemin $D u^{*, 1}$

${ }^{1}$ Institute of Biomedical \& Health Engineering, Shenzhen Institutes of Advanced Technology (SIAT), Chinese Academy of Sciences (CAS), Shenzhen, China.

${ }^{2}$ State Key Laboratory of Supramolecular Structure and Materials, College of Chemistry, Jilin University, Changchun, China.

*E-mail: xm.du@siat.ac.cn. (X. D.)

\section{SUPPLEMENTARY FIGURES}

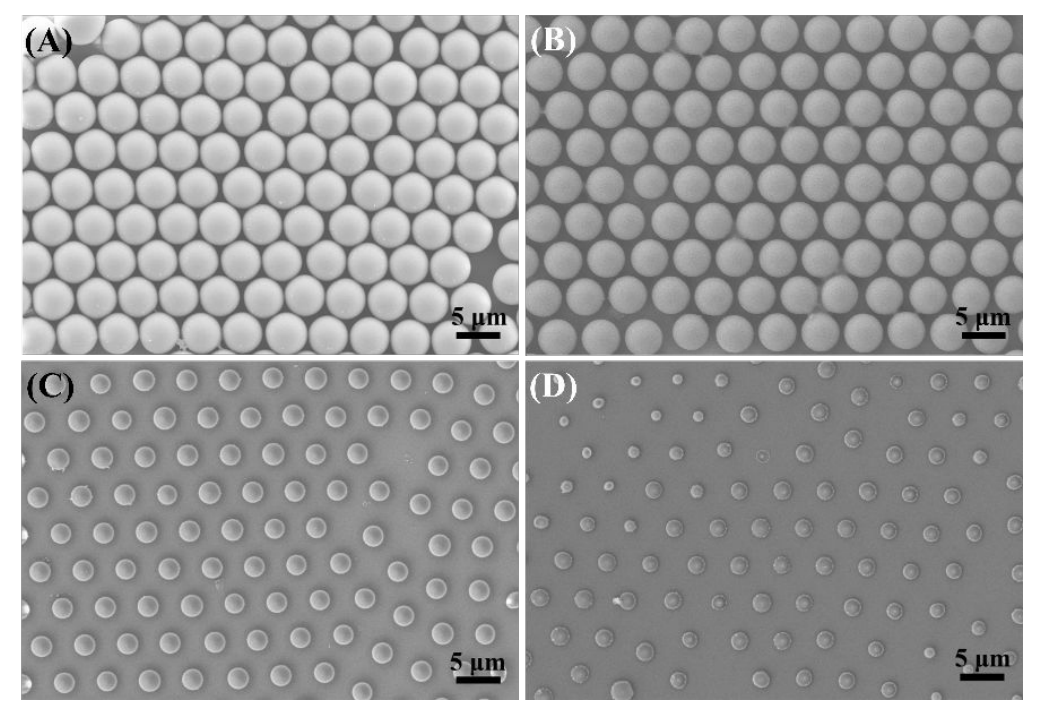

Figure S1. SEM images of PS microsphere array with the increasement of etching time. (A) Typical SEM image of hexagonal-close-packed polystyrene colloidal monolayer. (B-D) The SEM images of PS microspheres etched for $2 \mathrm{~min}, 4 \mathrm{~min}$ and $6 \mathrm{~min}$, respectively. The diameter of PS microsphere mask decreases gradually with the increasement of etching time. 

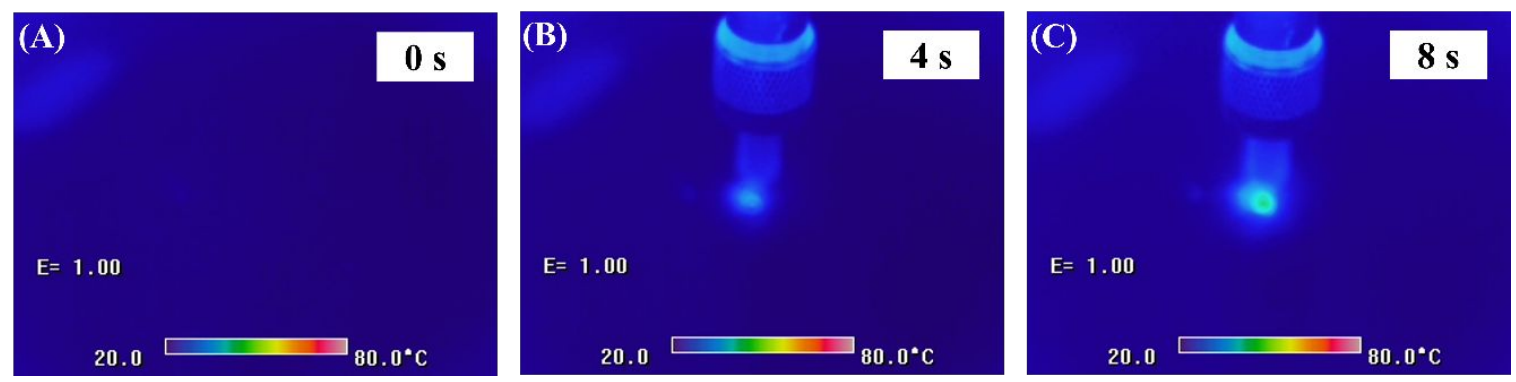

Figure S2. Infrared thermal images of the flat gold film without hollow microcone structure under NIR irradiation of $0 \mathrm{~s}(\mathrm{~A}), 4 \mathrm{~s}(\mathrm{~B})$ and $8 \mathrm{~s}(\mathrm{C})$. The flat gold film reaches $35^{\circ} \mathrm{C}$ within $8 \mathrm{~s}$ under NIR irradiation.

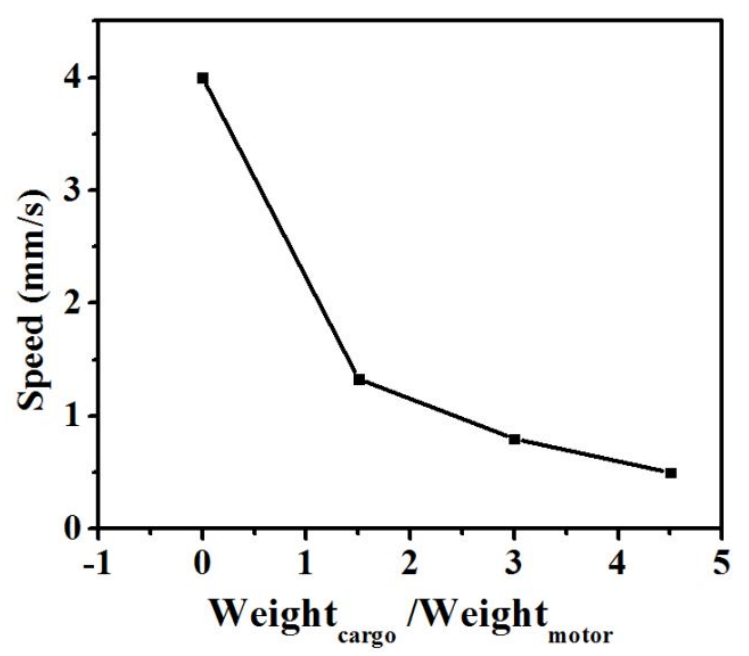

Figure S3. The AuHMA film with a size of $0.75 \mathrm{~cm} \times 0.75 \mathrm{~cm}$ can carry maximum 4.5 folds of its own weight. Decreasing the weight of the cargo increases the floating velocity.
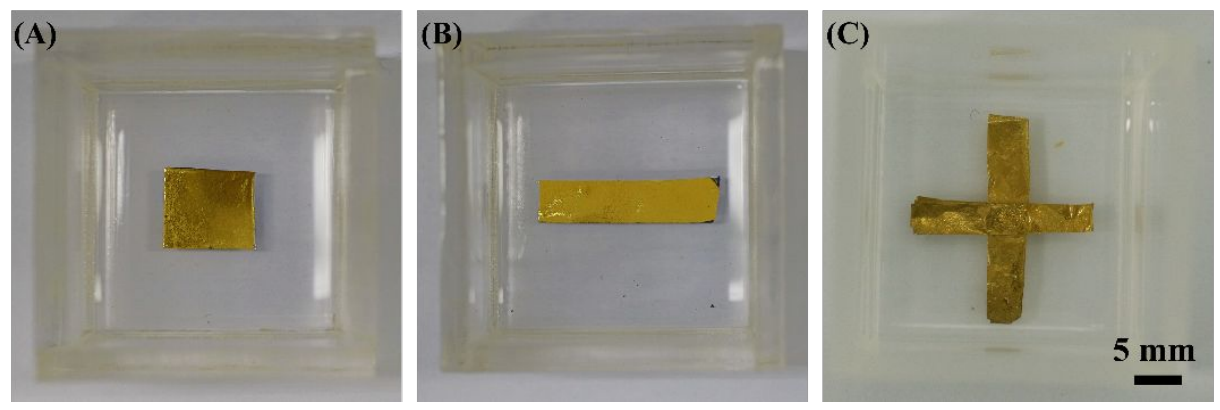

Figure S4. The optical photographs of top view of square AuHMA film (A), rectangular AuHMA film (B), cross-shaped AuHMA film (C). 

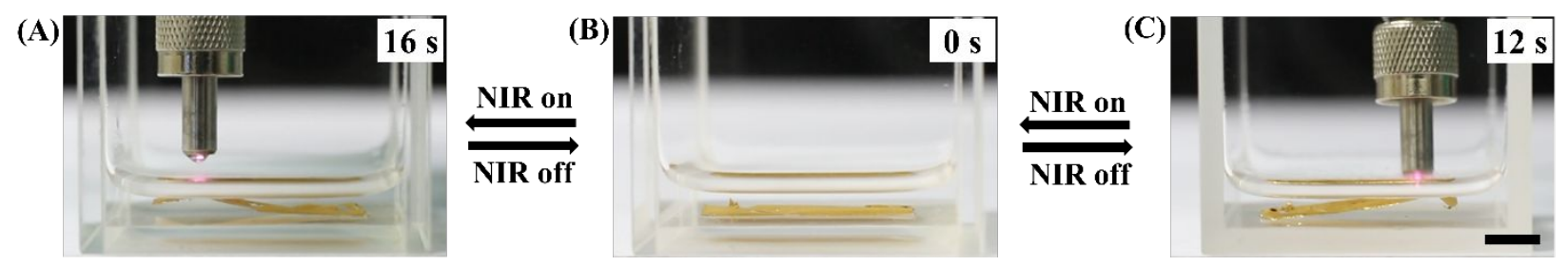

Figure S5. NIR laser spot irradiated the left side or right side of rectangular AuHMA film, the corresponding positions upward buckling within several seconds. Scale bar: $0.5 \mathrm{~cm}$.

\section{Supporting Movies}

Movie S1: NIR light-driven floating-diving motions of a AuHMA film in ethanol.

Movie S2: The floating-diving process of a blue film-loaded AuHMA film by the NIR on/off in ethanol.

Movie S3: The bending motions of the left side and right side of the rectangular AuHMA film by NIR irradiating at different positions.

Movie S4: NIR light-driven floating process of the rectangular AuHMA film in ethanol.

Movie S5: NIR light precisely controlled the completely floating of the AuHMA film with a complex morphology under the successive NIR irradiation the center and the sides of the AuHMA film.

Movie S6: The behavior of the cross shaped AuHMA film under NIR irradiation with low intensity.

Movie S7: Jellyfish-like floating motion of the cross shaped AuHMA film in ethanol.

Movie S8: A AuHMA film acted as a motor to drive a foam craft on the water surface as exposure to NIR irradiation. 